\title{
Eddy Current Measurement of the Electrical Conductivity and Porosity of Metal Foams
}

\author{
Xiandong Ma and A. J. Peyton
}

\begin{abstract}
This paper presents experimental results characterizing the electrical properties of metallic foams, a relatively new class of material, using nondestructive eddy current sensing techniques. The fundamentals of eddy current sensing, which is based on electromagnetic induction, are described, and the effects on coil impedance change of the representative types of coil sensors are analyzed. It has been found that the phase-frequency response of the normalized eddy current signal of the sensor is relatively immune to coil-to-sample spacing and fill-factor variations, from which key results such as the equivalent conductivity and the porosity of the foams are presented. The paper demonstrates the broad applicability of this technique in characterizing and further recognizing the properties of a variety of sample shapes used.
\end{abstract}

Index Terms-Coils, eddy currents, electromagnetic induction, impedance measurement, metallic foams.

\section{INTRODUCTION}

$\mathbf{C}$ ELLULAR metal foams are a relatively new class of material, which are of interest because of the exceptional ability to adapt their mechanical, thermal, acoustic, electrical, and chemical properties by varying their physical compositions such as porosity (relative density), geometry of the pores (shape, orientation, and size), and cell topologies (open-cell and closed-cell structure). Therefore, metallic foams offer many significant applications particularly in energy absorption, thermal management, lightweight structures, and automotive industry. There are wide ranges of manufacturing methods for metal foams. In terms of precursory forms and the types of pore-forming agents, typical methods can be grouped into melt-gas injection, melt-foaming agent, powder-foaming agent, pressure infiltration, and sintering-dissolution processes [1]. One interesting topic is to study the electrical properties of foams, which require nondestructive measurement. Eddy current sensing satisfies this need and furthermore enables improved control of metal foam processing through characterizing its electrical properties.

Eddy current methods have been used over several decades for nondestructive material evaluation and inspection [2]-[4]. Passing an alternating current through an excitation coil generates a fluctuating magnetic field in its vicinity. This timevarying magnetic field induces eddy currents in the electrically

Manuscript received June 15, 2004; revised October 7, 2005. This work was supported by the U.K. Engineering and Physical Sciences Research Council (EPSRC).

The authors are with the School of Electrical and Electronic Engineering, University of Manchester, Manchester M60 1QD, U.K. (e-mail: Xiandong. Ma@manchester.ac.uk; Anthony.Peyton@manchester.ac.uk).

Digital Object Identifier 10.1109/TIM.2006.873549 conductive test sample, which in turn generate a secondary magnetic field. The interaction between two fields alters the distribution of the magnetic flux, resulting in an apparent change of the coil impedance. By measuring this coil impedance change, the nature of the material can be inferred and further recognized by correlating the coil impedance using analytical and/or experimental methods to the characteristic quantities of interest, such as electrical conductivity, magnetic permeability, coating layer thickness, etc.

This paper presents experimental results characterizing the principal properties of aluminum foams such as equivalent electrical conductivity and porosity by means of eddy current sensing techniques. The application of eddy current inspection techniques to metal foams is relatively new. The induced eddy currents flowing in the foam samples are affected significantly by the foam properties, and consequentially measurement of the impedance change on the sensing coil(s) permits the metal foams to be characterized.

\section{Eddy CurRent Sensing Methodologies}

\section{A. Diffusion Phenomenon}

Eddy currents induced inside the test sample by the applied magnetic field attenuate with depth below the test sample surface. This attenuation is mainly governed by the test object's electrical conductivity $\sigma$, magnetic permeability $\mu$, and the applied frequency $f$ for a given test geometry. The standard depth of penetration $\delta$ can be used to characterize this diffusion phenomenon, which, for plane geometry, is mathematically given by

$$
\delta=\frac{1}{\sqrt{\pi f \mu \sigma}}
$$

where for a nonmagnetic electrically conductive foam the value of $\mu$ virtually equals to the permeability of free space, i.e., $4 \pi \times 10^{-7} \mathrm{H} / \mathrm{m}$. Apparently, high frequency measurements give information regarding the properties adjacent to the surface whereas low frequency testing probes the subsurface deeper inside the test sample. Multifrequency testing has been therefore employed.

\section{B. Coil Impedance}

The physical principle for electromagnetic induction problems can be described as a diffusion equation in terms of 


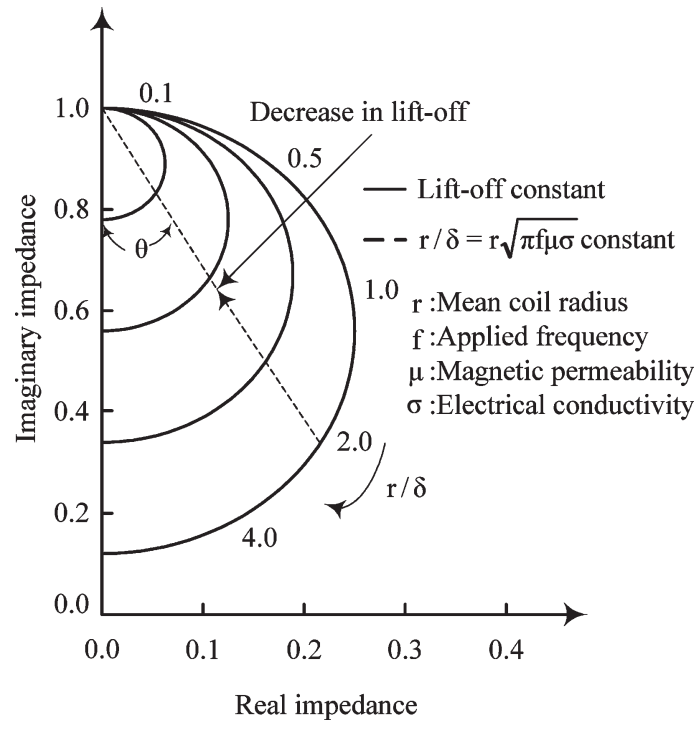

Fig. 1. Normalized impedance curves for planar circular coil varying with reference numbers $r / \delta$ (coil radius/skin depth) and lift-off (coil-to-sample spacing).

magnetic vector potential $\mathbf{A}$ as given in a complex phasor notation for the sinusoidal waveform excitation cases by

$$
\nabla^{2} \mathbf{A}+j \omega \mu \sigma \mathbf{A}=-\mu \mathbf{J}
$$

where $\mathbf{J}$ denotes the source current density passing through the excitation coil and $\omega$ is the angular frequency. This equation describes the penetration of eddy currents into material. Naturally, the solution of $\mathbf{A}$ must account for the necessary boundary conditions between the coil geometry and test materials. The electric field intensity $\mathbf{E}$ can be calculated through $\mathbf{E}=-\partial \mathbf{A} / \partial t$. The induced voltage in sensing coil is then computed by taking the line integral of the vector $\mathbf{E}$ around the coil loop and the coil impedance thus obtained.

The normalized impedance analysis is widely preferred for the analysis of eddy current signals in a complex-plane diagram. The normalized impedance is defined as the ratio of the measurement coil impedance due to the presence of the test object and the coil impedance as measured in the air, which provides the relative magnitude of eddy current signal with regards to background measurement. Dodd and Deeds [5] gave the analytical solution to a circular coil of rectangular cross section above a two-conductor plane. Fig. 1 illustrates the variations of real and imaginary impedance components for electrically conductive (nonmagnetic) materials under different values of reference number and lift-off (coil-to-sample spacing). The reference number is defined as $r / \delta$, i.e., the ratio of mean coil radius $r$ and skin depth. As is well known, the resistive (real) component of the coil impedance is related to energy losses caused by eddy currents within the test material. The magnetic field set up by the eddy currents tends to weaken the energizing coil field, thus lowering the value of the reactive (imaginary) component. This finally results in a comma-shaped normalized impedance diagram. As can be seen from this illustration, the reference number controls the data points shifting along the curve. The effect of decreasing lift-off causes the impedance curve to shrink, the smaller the value of lift-off, the closer the impedance curve approaches to the empty coil impedance point $(0, j)$. Apparently, the overall magnitude of eddy current signal is lift-off dependent, which means the magnitude is dependent on the test geometry. However, the phase angle $\theta$ is virtually lift-off invariant, which means phase signature is only dependent on the sample under test.

Libby [6] also reported the analytical solution to an infinitely long solenoid encircling a long cylindrical conductor within it. The comma-shaped loci, as shown in Fig. 1, also apply to eddy current sensing using solenoidal coils, where it is the reference number $r \sqrt{\omega \mu \sigma}$ and fill-factor that determine the variation of impedance curves. The fill-factor is defined as the ratio of the radii of test object and sensing coil. The fill-factor governs the effectiveness of the coupling energy into the test object, which affects the impedance loci in a same way as the lift-off effect does in cases of planar coils.

\section{Equivalent Electrical Conductivity}

The phase signature lift-off invariance is significant in that it indicates the existence of a relationship between phase angle $\theta$ and the reference number. For the block and sandwichshaped samples under test using planar pancake coils, a linear relationship has been observed between the cotangent of the phase angle and the reference number $r / \delta$ through a least square fit to the measured data of bulk materials [7]

$$
\cot \theta=b+a \frac{r}{\delta} .
$$

The electrical conductivity of the material $\sigma$ is thus solved in the value of

$$
\sigma=\frac{(\cot \theta-b)^{2}}{\pi \mu a^{2} r^{2} f} .
$$

The values of parameters $a$ and $b$ in (3) and (4) are application dependent mainly on the coil sensor geometry and can be calibrated using the measurement data of bulk materials.

For the cylindrically shaped samples under test using solenoidal coils, the relationship can be described parametrically by expressing the reference number and phase angle $\theta$ alone as

$$
\frac{1}{r \sqrt{\omega \mu \sigma}}=X(\theta) .
$$

The electrical conductivity $\sigma$ can be evaluated using the precalculated values of $X(\theta)$ by

$$
\sigma=\frac{1}{\omega \mu(r X(\theta))^{2}}
$$

where $X(\theta)$ can be calibrated based on the impedance curves of bulk materials as their electrical conductivity $\sigma$ is already known.

\section{Porosity Evaluation}

The mean porosity $\xi$ of foam is defined as $\left(1-\rho / \rho_{s}\right)(\%)$, where $\rho / \rho_{s}$ is the relative density defined as the density of 
foam $\rho$ divided by the density of solid $\rho_{s}$ of which it is made. Actually, the mean porosity can be obtained by weighting the foam and then dividing the weight of foam by that of solid in percentage with the same external dimensions. For closed-cell foams, the electrical conductivity is connected with porosity (relative density) for porous metal foams in the following relationship [8], which takes into account the fact that the equivalent electrical conductivity decreases with the increase in porosity

$$
\sigma=\frac{2 K(1-\xi)}{2 K+\xi} \sigma_{s}
$$

where $\sigma_{s}$ is the electrical conductivity of solid, $K$ is a constant determined by cell shape, and $K=0.3403$ when the cell shape is spherical. Thus, if the equivalent conductivity is known, the mean porosity of the foams can be estimated through

$$
\xi=\frac{0.6806\left(\sigma_{s}-\sigma\right)}{\sigma+0.6806 \sigma_{s}} .
$$

\section{Metallic Foams And Coil Designs}

\section{A. Aluminum Foams}

Sandwich-shaped and rod-shaped foams with a closed-cell structure were investigated in the measurements. Sandwichshaped foams were manufactured using $\mathrm{CaCO}_{3}$ as the foaming agent [9], which were produced using an Al-Si alloy with $10 \mathrm{vol} . \%$ of $\mathrm{SiC}$ particles ( $13 \mu \mathrm{m}$ in size) as a stabilizer. Three such foams were used as test samples, each having a size of $47.0 \mathrm{~mm} \times 42.7 \mathrm{~mm}$, but $4.5,9.2$, and $14.6 \mathrm{~mm}$ in thickness, respectively. The sandwich-shaped foams under test all have a porosity of approximately $85 \%$ and a pore size of about $1.5 \mathrm{~mm}$. In addition, two commercial foams were used in the testing. Alporas foam has a surface area of $149.9 \mathrm{~mm} \times 100.1 \mathrm{~mm}$ and $10.5 \mathrm{~mm}$ in thickness, whereas Cymat foam has a surface area of $102.4 \mathrm{~mm} \times 49.7 \mathrm{~mm}$ and $53.0 \mathrm{~mm}$ in thickness. They have approximately same porosity of about $90 \%$ but with different pore sizes ( $3 \mathrm{~mm}$ for Alporas and $6 \mathrm{~mm}$ for Cymat foam).

Rod-shaped foams were produced by a powder route [1]. Two aluminum rod foams with different porosities were used as the rod-shaped samples. The porosities by weight are $71.83 \%$ and $80.41 \%$ for rod foam 1 and rod foam 2, respectively. Both foams are $10 \mathrm{~mm}$ in diameter and $200 \mathrm{~mm}$ in length and have a pore size of around $1.0 \mathrm{~mm}$.

\section{B. Coil Geometries}

Two types of sensor coils have been constructed purposely to accommodate different shaped samples, which are based on planar pancakes and solenoidal windings. Planar pancakes are used to inspect block and sandwich foams, whereas solenoids are used to test cylindrically shaped rod samples. Fig. 2 shows the PCB pancake coil and solenoidal winding geometries, respectively, used for the applications, where the geometry parameters are described in Table I. In practical measurements, double-coil arrangements are preferred, where one coil is used for excitation purposes with a separate secondary coil used for
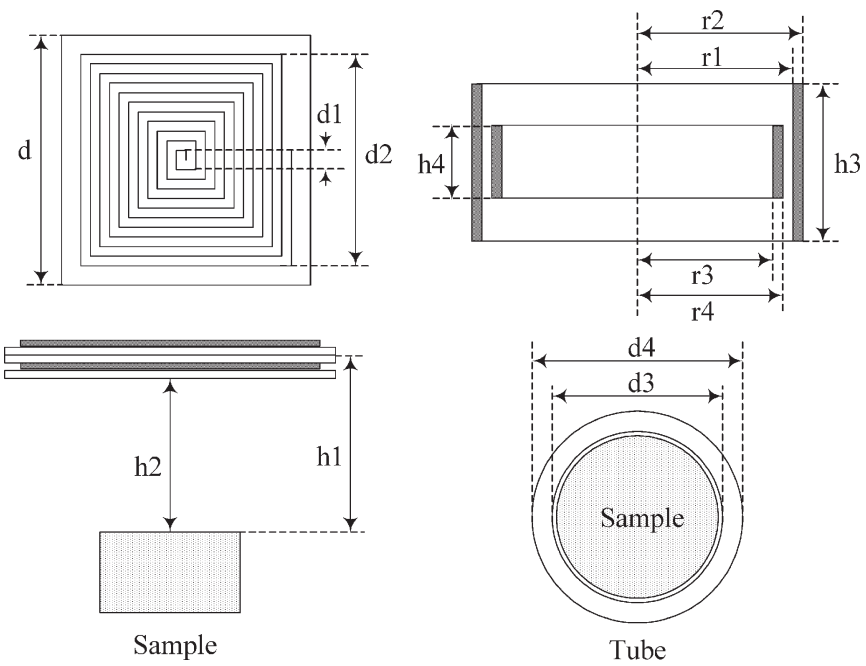

Fig. 2. Coil sensors used in the applications. (Left) Planar pancake coils. (Right) Solenoidal sensor.

TABLE I

PARAMETERS OF SENSOR GEOMETRY

\begin{tabular}{lc}
\hline Parameters & Quantity $(\mathrm{mm})$ \\
\hline PCB pancake coils & \\
d: PCB board size & 59.0 \\
d1: Initial square size & 11.0 \\
d2: Final square size & 41.0 \\
h1: Excitation coil-to-sample spacing & \\
h2: Sensing coil-to-sample spacing & \\
h1-h2: Separation between coils & 3.0 \\
Number of turns of excitation coil & 16 \\
Number of turns of sensing coil & 16 \\
& \\
Solenoidal coils & \\
r1: Inner radius of excitation coil & 11.0 \\
2: Outer radius of excitation coil & 11.25 \\
r3: Inner radius of sensing coil & 10.5 \\
r4: Outer radius of sensing coil & 10.75 \\
h3: Excitation coil height & 12.0 \\
h4: Sensing coil height & 6.0 \\
d3: Inner diameter of tube & 11.0 \\
d4: Outer diameter of tube & 21.0 \\
Number of turns of excitation coil & 40 \\
Number of turns of sensing coil & 20
\end{tabular}

sensing. The excitation coil is used to generate a fluctuating magnetic field whereas the sensing coil, placed concentrically with the excitation coil, is used to detect the perturbation of the primary field resulting from the presence of test sample. The resonant frequency of the sensor is in the region of 2 and $3 \mathrm{MHz}$ for solenoidal and pancake coils, respectively.

\section{EXPERIMENTAL RESULTS}

A Solartron SL 1260 impedance analyzer was used in the tests, which can supply a sinusoidal signal with variable frequencies to the excitation coil of the sensor. The analyzer recorded the ratios of the induced voltage across the sensing coil and the current flowing through the excitation coil, obtaining real and imaginary parts of the gain and phase of the transfer 

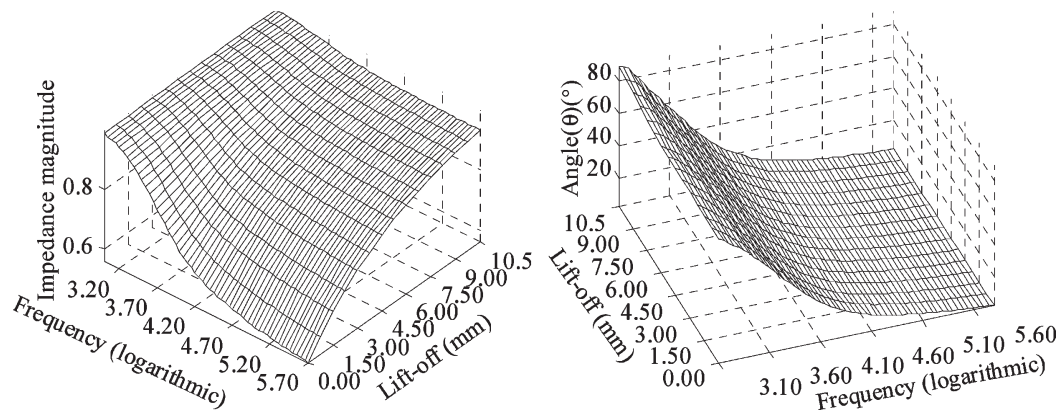

Fig. 3. Lift-off effect on the eddy current signal. (Left) Impedance magnitude and (right) phase signature, where coil-to-sample spacing ranges from 1.5 to $10.5 \mathrm{~mm}$ and the overall swept frequencies are between 1.25 and $630 \mathrm{kHz}$.

impedance of the sensor. Coil impedance measurements in the air were first made to normalize impedance calculations.

The selection of an effective operation frequency range is normally critical, and this depends largely on the coil configuration and the properties of the foam samples. The selection of lower operating frequency is largely dependent on the thickness or radius of the test sample and is limited by signal to noise performance of the measurement system due to the inductive nature of the sensor. The electromagnetic skin depth at this frequency should be comparable with the thickness or radius of the sample to ensure that a sufficient volume of material is probed. Lowering further the test frequency reduces the effectiveness of the coupling energy into the test object considerably, thus resulting in low sensitivity measurements. In contrast, the upper operating frequency should be selected at which the electromagnetic skin depth exceeds the pore size of the foams so that the impact of pore size of the foams can be fully considered. The measurements can be made with a relatively good accuracy at these intermediate frequencies ranging from the selected lower frequency to the upper frequency. Take the sandwich foam with a thickness of $9.2 \mathrm{~mm}$ and a pore size of $1.5 \mathrm{~mm}$ as an example. Substituting $\delta=9.2 \mathrm{~mm}$ into (1) gives a lower frequency of around $600 \mathrm{~Hz}$, for instance, when $\sigma=5 \mathrm{MS} / \mathrm{m}$. Supposing a much lower conductivity of foams compared to that of bulks, $1.5 \mathrm{kHz}$ is selected as the lower operating frequency in practical calculations. Considering the pore size of $1.5 \mathrm{~mm}$ of this foam, the upper operating frequency should be in the region of $50 \mathrm{kHz}$, supposing the conductivity of the porous foam is lower than $3 \mathrm{MS} / \mathrm{m}$, which is obviously far less than the resonant frequency of $3 \mathrm{MHz}$ of the pancake coils.

\section{A. Sandwich-Shaped Foams}

Sandwich-shaped foams were examined with the planar pancake coil arrangement as shown in Fig. 2. As described earlier, impedance curves are highly affected by the lift-off. Fig. 3 illustrates the variations of eddy current signal with liftoff and applied frequency in three-dimensional (3-D) form. In this example, a sandwich foam $4.5-\mathrm{mm}$ thickness is shown, which describes the responses of impedance magnitude and phase angle $\theta$ to both the coil-to-sample spacing ranging from 1.5 to $10.5 \mathrm{~mm}$ and the overall swept frequencies between 1.25 and $630 \mathrm{kHz}$ applied at each distance. This demonstrates again that impedance magnitude varies with the lift-off sig-

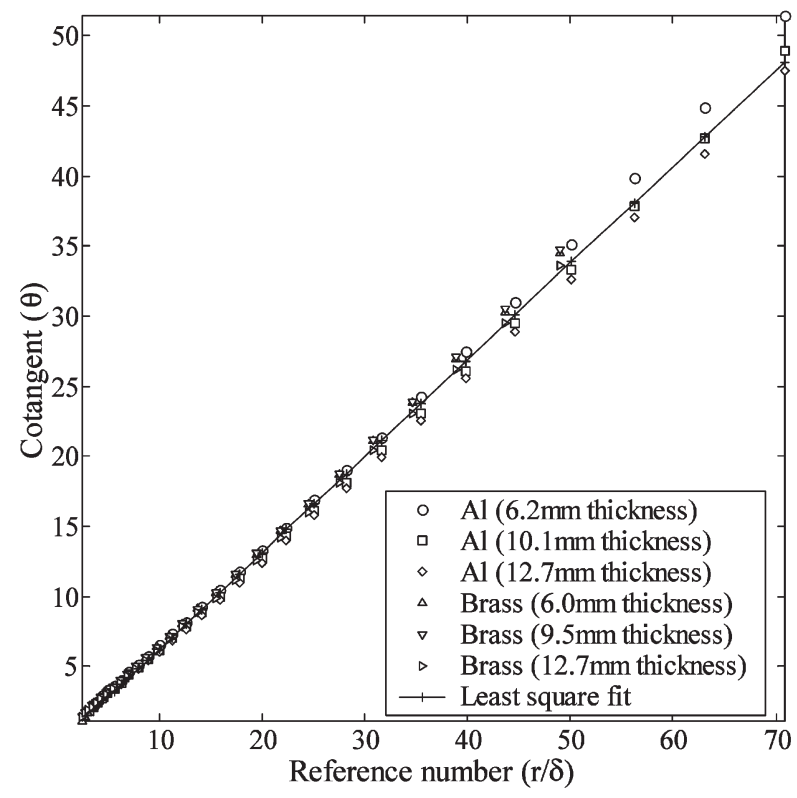

Fig. 4. Cotangent of phase angle against reference number (coil radius/skin depth) for a variety of bulk materials under pancake coils.

nificantly; however, the phase signature is lift-off invariant. Phase signature is therefore interested in characterizing eddy current signal as it is only related with the material under test as described earlier.

Three solid aluminum sandwiches and three solid brass sandwiches with similar sizes to the foams were tested to calibrate the sensor coils as their electrical conductivity $\sigma$ is already known $(\sigma=37.67 \mathrm{MS} / \mathrm{m}$ for aluminum and $\sigma=18.0 \mathrm{MS} / \mathrm{m}$ for brass). Fig. 4 gives the relationship between phase signature and reference number based on the measured data of bulk materials, where $x$-axis represents the reference number $r / \delta$, which is proportional to the square root of operation frequency, and $y$-axis as the cotangent of the phase angle. A straight-line relationship was observed between two parameters for different bulk materials with a variety of thickness. This can be numerically fitted through a least square fit to the measured data, obtaining $a=0.6932$ and $b=-0.5545$ in (3). Hence, the calculation of electrical conductivity can be achieved in terms of (4), as shown in Fig. 5. In this group test, the frequency range best suitable for the foam with the intermediate thickness $(9.2 \mathrm{~mm})$ as discussed earlier is taken as the compromise, namely, the low and upper operating frequencies 


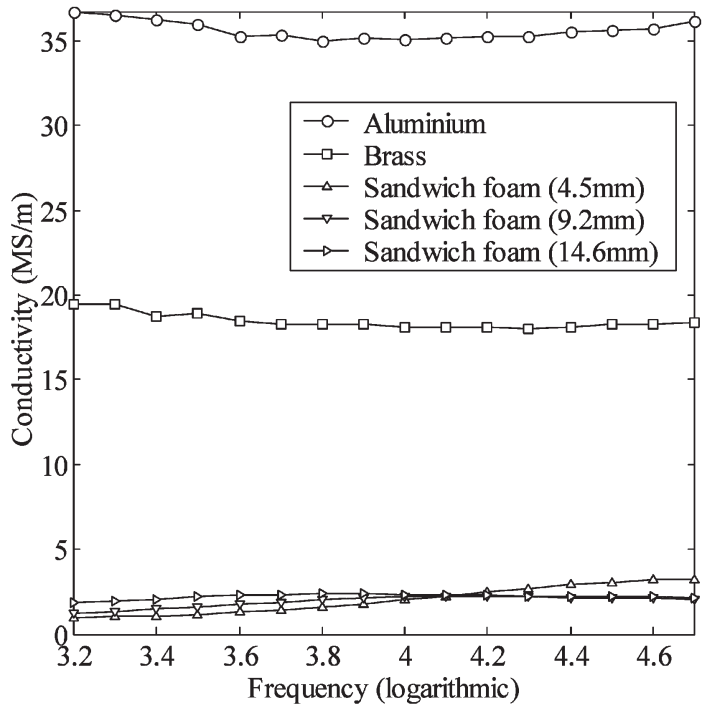

Fig. 5. Values of electrical conductivity for sandwich-shaped foams and bulk materials, where the effective frequency range is between 1.5 and $50.0 \mathrm{kHz}$.

TABLE II

Values of Porosity Measured by Weight and Those Measured USING EDDY CURRENT SENSING TECHNIQUES FOR THE ALUMinum FoAms TEST

\begin{tabular}{lcc}
\hline Foam & $\begin{array}{l}\text { Porosity by } \\
\text { weight (\%) }\end{array}$ & $\begin{array}{l}\text { Measured } \\
\text { porosity (\%) }\end{array}$ \\
\hline Sandwich shaped foam $(4.5 \mathrm{~mm})$ & 85.0 & 86.28 \\
Sandwich shaped foam $(9.2 \mathrm{~mm})$ & 85.0 & 88.29 \\
Sandwich shaped foam $(14.6 \mathrm{~mm})$ & 85.0 & 86.72 \\
Alporas foam & 89.94 & 79.91 \\
Cymat foam & 90.05 & 85.67 \\
Rod shaped sample 1 & 71.83 & 72.12 \\
Rod shaped sample 2 & 80.41 & 79.56 \\
\hline
\end{tabular}

are 1.5 and $50 \mathrm{kHz}$, respectively. The values of equivalent conductivity of the foams by taking a mean value within this effective frequency range are about $2.28,1.92$, and $2.20 \mathrm{MS} / \mathrm{m}$, respectively. The values of solid aluminum and brass are also displayed in the illustration as the reference in order to show the relative magnitude of electrical conductivity of the foams. The measured electrical conductivities of bulk aluminum and brass are 35.62 and $18.40 \mathrm{MS} / \mathrm{m}$, respectively, which implies that the measurement error is within 5\%. Table II gives the values of porosity measured with the eddy current sensing technique in comparison with those obtained by weight. The measurement results for porosity are in good agreement with those actual mean porosities measured by weight.

Now consider the application of this sensing technique to commercial Alporas and Cymat aluminum foams. A relatively large pancake coil sensor was constructed owing to the significant increasing dimension of the foam samples. In fact, this large pancake coil sensor has the same test arrangement as the pancake coils shown in Fig. 2 but with the geometry parameters being chosen as: PCB board size $d=125 \mathrm{~mm}$, initial square $d 1=17 \mathrm{~mm}$, final square $d 2=103 \mathrm{~mm}$, and the number of excitation/sensing coil is 35 turns. The relationship between the cotangent of the phase signature and the reference number

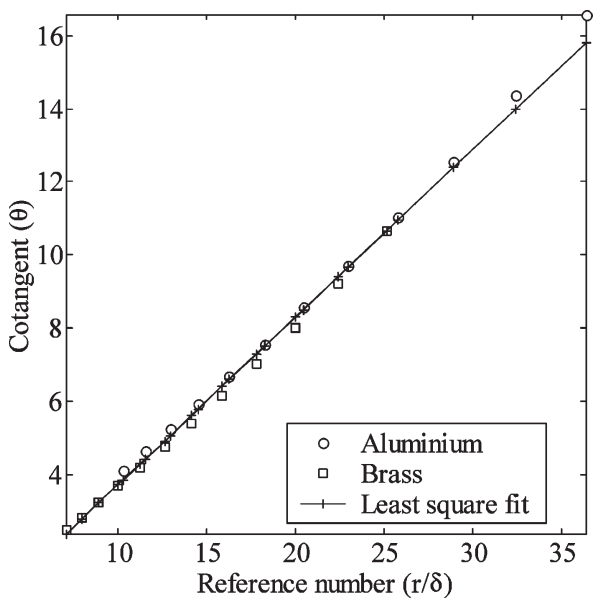

Fig. 6. Cotangent of phase angle against reference number (coil radius/skin depth) for bulk materials under large pancake coils.

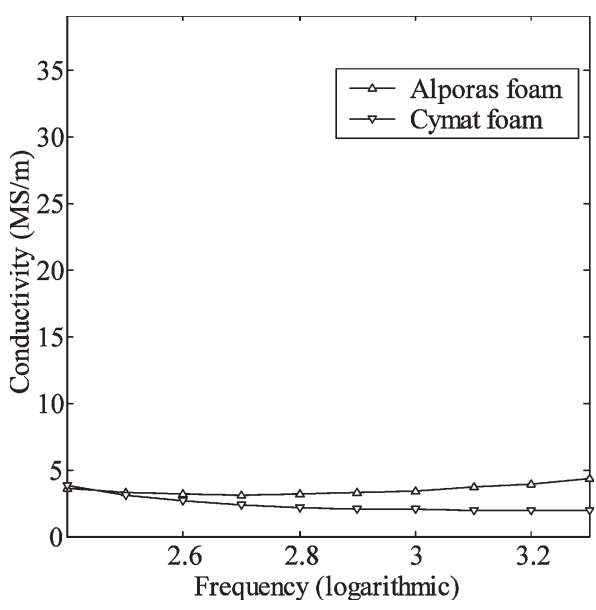

Fig. 7. Values of electrical conductivity for commercial foams where the effective frequency range is between $250 \mathrm{~Hz}$ and $2.0 \mathrm{kHz}$.

based on the measured data of bulk materials is shown in Fig. 6, from which the parameters $a=0.4584$ and $b=-0.8819$ are obtained. It is worth noting that a relatively lower frequency $(250 \mathrm{~Hz})$ was chosen in this case due to the relatively large thickness of these foams and, at frequencies below $250 \mathrm{~Hz}$, the measurement data became noisy because of the inductive nature of the sensor. Meanwhile, the value of upper frequency $(2.0 \mathrm{kHz})$ is relatively small; this is mainly attributed to the large pore size that commercial foams have (3 $\mathrm{mm}$ for Alporas and $6 \mathrm{~mm}$ for Cymat foam). Fig. 7 shows the electrical conductivity of foams under an effective operation frequency between $250 \mathrm{~Hz}$ and $2.0 \mathrm{kHz}$. On this basis, the deduced equivalent conductivities are 3.48 and 2.395 MS/m for Alporas and Cymat foams, respectively. There is a relatively big difference between the measurement porosities and the actual mean porosities for the commercial foams as shown in Table II. The Alporas and Cymat foams under test were found to have specific structures such as the same material coatings on the foam surfaces. This increases the relative density of the test foams due to solid surface, thus increasing the measured equivalent conductivity, which finally leads to the decreased mean porosities when 


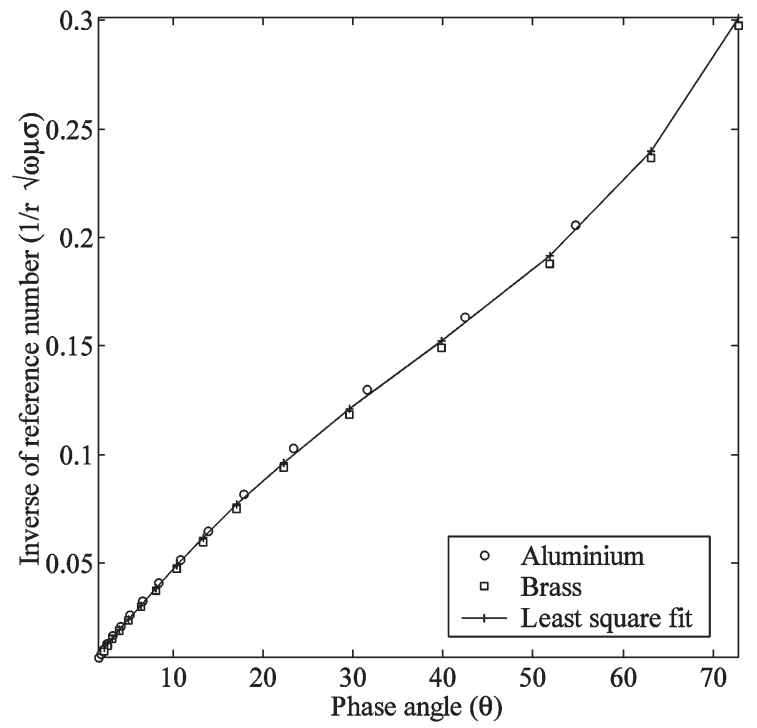

Fig. 8. Inverse of reference number $r \sqrt{\omega \mu \sigma}$ against the phase angle $\theta$ for bulk materials under solenoidal coils.

calculated in terms of (8). These factors result in discrepancies of the measurement results.

\section{B. Rod-Shaped Foams}

In the same procedure, the relationship between reference number and phase angle was first built up prior to conductivity evaluation. In Fig. 8, values of the inverse of reference number $r \sqrt{\omega \mu \sigma}$ are plotted against the phase angle $\theta$ for bulk aluminum and brass materials of similar sizes to rod foams. A polynomial expression can be used to best fit numerically to these measured data, i.e.,

$$
\begin{aligned}
X(\theta)=-0.0018 & +0.0053 \theta-3.6852 \times 10^{-5} \theta^{2} \\
- & 2.6145 \times 10^{-7} \theta^{3}+7.6855 \times 10^{-9} \theta^{4} .
\end{aligned}
$$

Substituting $X(\theta)$ into (6) enables the electrical conductivity to be calculated.

Fig. 9 illustrates the conductivity curves within the effective frequency range between 1.0 and $50.0 \mathrm{kHz}$, which are determined by the radius of the foams and the pore size, respectively. Values of equivalent conductivity are finally computed as follows: $5.10 \mathrm{MS} / \mathrm{m}$ for rod foam 1, $3.55 \mathrm{MS} / \mathrm{m}$ for rod foam 2, 35.75 MS/m for solid aluminum rod, and $16.5 \mathrm{MS} / \mathrm{m}$ for solid brass rod. Porosities of rod foams have been obtained from a relationship between electrical conductivity and porosity as described in (7) and (8), and are also in good agreement with those obtained by weight as given in Table II.

\section{CONCLUSION}

This study has shown that eddy current techniques can be effectively used to examine the electrical properties and further the structure of porous aluminum foams. Typical coil sensors (planar pancake coils and solenoidal windings) have been designed to accommodate the representative types of

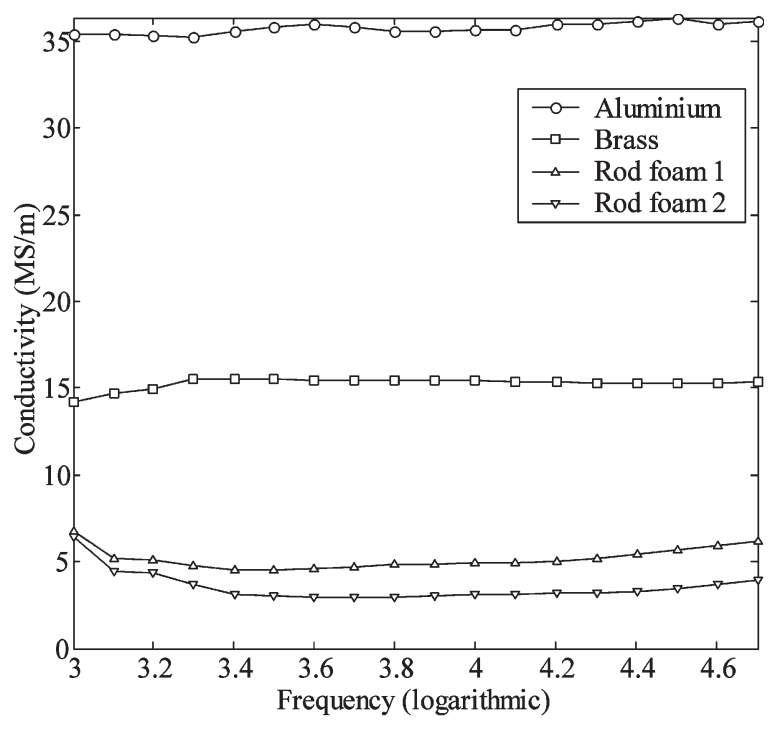

Fig. 9. Values of electrical conductivity for rod foams and bulk materials, where the effective frequency range is between 1.0 and $50.0 \mathrm{kHz}$.

sample shapes (sandwich-shaped and rod-shaped foams). It has been found that the phase-frequency response of the sensor is relatively immune to coil-to-sample spacing and fill-factor variations, from which the equivalent electrical conductivity of the sample can be determined. The equivalent conductivity can be converted into structural parameters such as mean porosity. The appropriate selection of an effective operation frequency range is critical in achieving reliable results, which depends mainly on the coil configuration and the properties of the foam sample. The lower operating frequency should be selected at which the electromagnetic skin depth is comparable with the thickness or radius of the sample to ensure that a sufficient volume of material is probed. The upper operating frequency should be selected at which the electromagnetic skin depth exceeds the pore size of the foams so that the impact of pore size of the foams can be fully considered. The research has demonstrated the potential of using this nondestructive testing technique in characterizing and further recognizing the properties of metallic foams.

Further work includes analytical forward problem solution for coil impedance change due to the presence of foams. The work also includes metallic foam eddy current simulations using the finite-element method to fully investigate the impact of the macrostructures of the foams on their electrical properties and further to establish a more accurate relationship between the equivalent electrical conductivity and the mean porosity. It is believed that the application of eddy current sensing holds very significant promise for the future industrial process in metallic foams.

\section{ACKNOWLEDGMENT}

The authors wish to thank Dr. V. Gergely and Mr. D. Curran of the Department of Materials Science and Metallurgy, Cambridge University, for providing the aluminum foams contributed in the measurements. This work was performed at Lancaster University, U.K. 


\section{REFERENCES}

[1] J. Banhart, "Manufacture, characterisation and application of cellular metals and metal foams," Prog. Mater. Sci., vol. 46, no. 6, pp. 559-632, 2001.

[2] L. Bavall, "Determination of the thickness of copper coatings on steel by measuring the impedance of a thin elliptic coil," Meas. Sci. Technol., vol. 13, no. 4, pp. 510-519, 2002.

[3] B. de Halleux, B. de Limburg Stirum, and A. Ptchelintsev, "Eddy current measurement of the wall thickness and conductivity of circular nonmagnetic conductive tubes," NDT E Int., vol. 29, no. 2, pp. 103-109, Apr. 1996.

[4] G. Mook, R. Lange, and O. Koeser, "Non-destructive characterisation of carbon-fibre-reinforced plastics by means of eddy-currents," Compos. Sci. Technol., vol. 61, no. 6, pp. 865-873, May 2001.

[5] C. V. Dodd and W. E. Deeds, "Analytical solutions to eddy-current probecoil problems," J. Appl. Phys., vol. 39, no. 6, pp. 2829-2838, May 1968

[6] H. L. Libby, Introduction to Electromagnetic Non-Destructive Test Methods. New York: Wiley, 1971.

[7] S. N. Vernon, "A single-sided eddy current method to measure electrical resistivity," Mater. Eval., vol. 46, no. 12, pp. 1581-1587, Nov. 1988.

[8] Y. Feng, H. Zheng, Z. Zhu, and F. Zu, "The microstructure and electrical conductivity of aluminum alloy foams," Mater. Chem. Phys., vol. 78, no. 1, pp. 196-201, 2003.

[9] V. Gergely, D. C. Curran, and T. W. Clyne, "The FOAMCARP process: Foaming of aluminium MMCs by the chalk-aluminium reaction in precursors," Compos. Sci. Technol., vol. 63, no. 16, pp. 2301-2310, Dec. 2003.

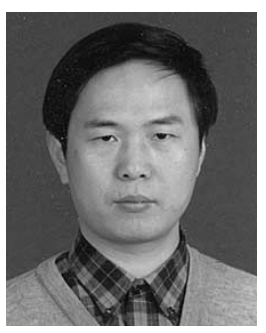

Xiandong Ma received the B.Eng. degree in electrical engineering from Jiangsu University of Science and Technology, China, in 1986, the M.Sc. degree in power systems and automation from Nanjing Automation Research Institute (NARI), China, in 1989, and the Ph.D. degree in partial discharge detection and analysis from Glasgow Caledonian University, U.K., in 2003.

From 1989 to 1998, he was an Electrical Engineer for eight years and later a Senior Engineer for two years at NARI, where he specialized in DSP-based power system real-time digital simulators dedicated for close-loop power equipment testing. From 2002 to 2004, he was a Research Associate at the Engineering Department, Lancaster University. He is currently with the University of Manchester, with research interests in the area of electromagnetic induction imaging for industrial process, nondestructive testing, and instrumentation.

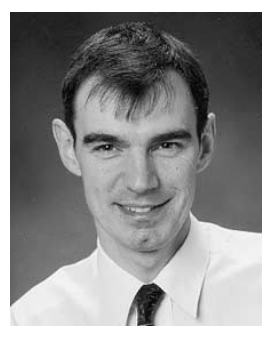

A. J. Peyton received the B.Sc. degree in electrical engineering and electronics and the Ph.D. degree in medical instrumention from the University of Manchester Institute of Science And Technology (UMIST), U.K., in 1983 and 1986, respectively.

In 1989, he was appointed as Principal Engineer at Kratos Analytical Ltd., developing precision electronic instrumentation systems for magnetic sector and quadrupole mass spectrometers, from which an interest in electromagnetic instrumentation developed. He returned to UMIST as a Lecturer and worked with the Process Tomography Group. In 1996, he moved to Lancaster University with his research team, taking up post as a Senior Lecturer. He was promoted to Reader in Electronic Instrumentation in July 2001 and Professor in May 2004, and returned to Manchester University in December 2004. His main current research interests are in the area of instrumentation, applied sensor systems, and electromagnetics. 